\title{
VHDL Realization of Efficient H.264 Intra Prediction Scheme Based on Best Prediction Matrix Mode
}

\author{
Sara Hamdy \\ Electrical Engineering Department \\ Benha Faculty of Engineering \\ Benha University - Egypt
}

\author{
Mostafa E. A. Ibrahim \\ Electrical Engineering Department \\ Benha Faculty of Engineering \\ Benha University - Egypt
}

\author{
Abdelhalim Zekry \\ Electronics and Communications Department \\ Faculty of Engineering \\ Ain Shams University - Egypt
}

\begin{abstract}
This paper introduces a VHDL realization of a new efficient intra prediction scheme that aims to enhance the compression efficiency of the H.264 standard. The new proposed algorithm is called Best Prediction Matrix Mode (BPMM). The main idea behind the new prediction scheme is to combine the most usable intra prediction modes, $\{$ vertical - horizontal - DC $\}$, into a new efficient prediction mode. The performance of the new proposed prediction scheme with respect to compression ratio, Peak Signal to Noise Ratio (PSNR) and bit rate is evaluated. The results show that the BPMM enhances the compression ratio and correspondingly the bit rate and it noticeably increases the PSNR.
\end{abstract}

Keywords:

Video Compression, H.264, Intra Prediction

\section{INTRODUCTION}

Video compression systems are used in many commercial products, from consumer electronic devices such as digital camcorders, cellular phones to video teleconferencing systems. These applications make the video compression hardware devices an inevitable part of many commercial products. In order to improve the performance of the existing applications, a new international standard for video compression which is named H.264 or MPEG4 Part-10 is developed. The new standard significantly improves video compression efficiency [1].

Figure (1) illustrates the main building blocks of the H.264 encoder. It is clear from the block diagram that the video compression efficiency of the H.264 standard is not a result of single feature but a combination of a number of encoding sub blocks. One of the most important factors of the improved compression efficiency of the H.264 is it's intra prediction algorithm [2]3]. An Intra prediction mode is the formation of a predicted block $\mathrm{P}$ based on previously encoded and reconstructed blocks and subtracted from the current block prior encoding. The predicted block $\mathrm{P}$ is formed for each $4 \times 4$ block or for a $16 \times 16$ macroblock $(\mathrm{MB})$. There are nine prediction modes available for $4 \times 4$ luma block, four modes for a $16 \times 16$ luma MB and four modes for the chroma blocks to remove spatial redundancy within a frame. The prediction mode for each block which results in minimum difference between $\mathrm{P}$ and the current block is selected. The first three prediction modes (Vertical, Horizontal,and DC) that are used for encoding intra $4 \times 4$ block are

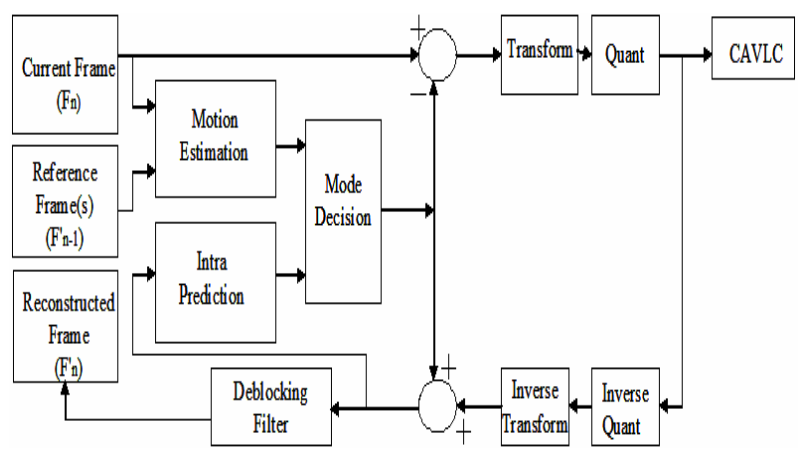

Fig. 1: Block Diagram of the H.264 Encoder

most commonly used, collectively they cover $85-95 \%$ of the best modes [4]

A lot of efforts have been made to improve the intra prediction scheme of H.264. Many research contributions have been done to simplify the intra coding in H.264. But it has been witnessed that in most of the cases the researchers have less contribution in improving coding efficiency [5 6].

In this paper, the authors are enthusiastic to propose a new efficient mode that combines the vertical, horizontal, and DC modes. The new proposed mode is titled BPMM. The performance of the BPMM is verified in terms of compression ratio, bit rate and PSNR. Furthermore, the efficiency of the BPMM is compared with other best known intra prediction algorithms.

The rest of the paper is organized as follows: Section 2 gives a theoretical background of the intra prediction in the H.264 encoder. Section 3 describes some of the commonly utilized intra prediction algorithms. Section 4 shows a comparison between the pre-described intra prediction algorithms to figure out the best algorithm regarding the compression ratio, PSNR and the bit rate. Section 5 explains the proposed new intra prediction approach. Section 6 illustrates the H.264 hardware encoder testbed that has been utilized to evaluate the proposed technique. In addition, it compares the best algorithm, decided before, with the achieved results of applying the new proposed prediction technique. Finally, Section 7 summarizes the main contributions of this paper. 


\section{H.264 INTRA PREDICTION OVERVIEW}

The main goal behind intra prediction is to achieve compression within a frame. The neighboring pixels within the picture frame tend to have similar values in order to exploit the spatial redundancy, the prediction is done based on the values of reconstructed pixels of previous sub block [1]. For the luminance layer intra frame prediction, there are two possible block sizes to encode one MB. The first is the I16MB, with four possible prediction modes applied to the whole $16 \times 16 \mathrm{MB}$. The second is the I4MB, with nine possible prediction modes applied to the sixteen blocks of $4 \times 4$ sub blocks which compose the MB. For the chrominance layer there are also four possible modes to predict each $8 \times 8$ block $(\mathrm{Cr}$ and $\mathrm{Cb})$ in $\mathrm{MB}$ [7, 8$]$.

The modes of the $4 \times 4$ intra prediction are given in Table 1 . The values of each $4 \times 4$ block of luma samples are predicted from the neighboring pixels above or left of a $4 \times 4$ block. Modes $0,1,3,4$, $5,6,7,8$ are directional ways of performing the prediction that can be selected by the encoder. However, mode 2 is the DC prediction mode with no direction [9].

Table 1. : Intra $4 \times 4$ prediction Mode

\begin{tabular}{cccc}
\hline Mode \# & Mode Name & Mode \# & Mode Name \\
\hline 0 & Vertical & 1 & Horizontal \\
2 & DC & 3 & Diagonal-Down-Left \\
4 & Diagonal-Down-Right & 5 & Vertical-Right \\
6 & Horizontal-Down & 7 & Vertical-Left \\
8 & Horizontal-Up & & \\
\hline
\end{tabular}

Figure (2a) shows $4 \times 4$ block containing 16 pixels labeled from 'a' through 'p'. A prediction block $\mathrm{P}$ is calculated based on the pixels labeled A-M obtained from the neighboring blocks [10]. Figure $(2 \mathrm{p}, \mathrm{c})$ show the intra H.264 standard $4 \times 4$ vertical and horizontal prediction modes [11]. If the prediction mode chosen is vertical, it means that each pixel value is vertically similar and the vertical edge is more probable than the horizontal edge in the block. In the same manner if the prediction mode chosen is horizontal, it means that each pixel value is horizontally similar and the horizontal edge is more probable than the vertical edge in the block [12, 13]. While in the DC prediction mode all pixels in the current $4 \times 4$ block are replaced by the mean value of the neighboring pixels $\mathrm{A}, \mathrm{B}, \mathrm{C}, \mathrm{D}, \mathrm{I}, \mathrm{J}, \mathrm{K}$ and $\mathrm{L}$. The DC prediction is accomplished by using the rules in Fig.(2d).

\section{INTRA PREDICTION ALGORITHMS}

In this section, the authors present two of the most utilized intra prediction algorithms. In addition, two other algorithms that up to the authors knowledge are new to this field are introduced.

\subsection{Sum of Absolute Differences (SAD)}

SAD is a widely used and extremely simple algorithm for measuring the similarity between image blocks. It works by taking the absolute difference between each pixel in original block and the corresponding pixel in the block being used for comparison as shown in Equation (1).

$$
S A D=\sum_{i=0}^{4} \sum_{j=0}^{4}|S(i, j)-C(i, j)|
$$

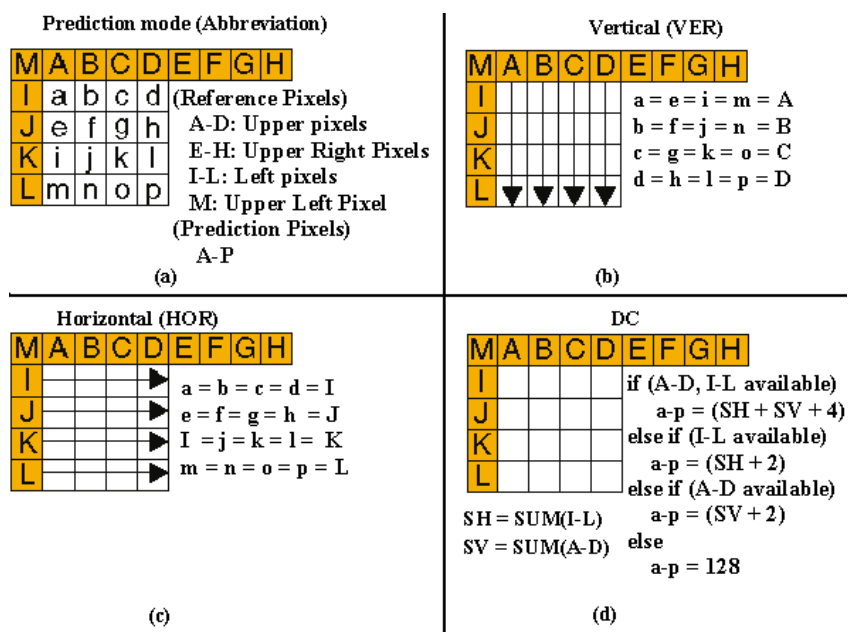

Fig. 2: Equations of luma $4 \times 4$ modes prediction pixels (Vertical, Horizontal,DC) reproduced from [11

where $S(i, j)$ and $C(i, j)$ are the $(i, j)^{t h}$ elements of the current original block $S$ and the reconstructed block $C[14,15]$.

\subsection{Sum of Squared Differences (SSD)}

SSD is the second widely utilized algorithm for measuring the similarity. It is the sum of squared difference between the original block $S$ and the reconstructed block $C$, and it is expressed by Equation [2] [16]:

$$
S S D=\sum_{i=0}^{4} \sum_{j=0}^{4}(S(i, j)-C(i, j))^{2}
$$

\subsection{Sum of Hamming Distances (SHD)}

Although, Hamming distance is an old technique for measuring the similarity, it is new to the field of intra prediction. Hamming distance is the number of positions at which the corresponding pixels are different. Equation (3) illustrates the way of computing the SHD.

$$
S H D=\sum_{i=0}^{4} \sum_{j=0}^{4}(S(i, j) \bigoplus C(i, j))
$$

where the symbol $\bigoplus$ denotes the bitwise XOR logic operation between the $(i, j)^{t h}$ elements of the current original block $\mathrm{S}$ and the reconstructed block $\mathrm{C}$.

\subsection{Manhattan Distance(MD)}

The Manhattan distance is also new to the intra prediction field. It is similar to the SAD except that in the MD the sum operation is divided into parts which allows smaller hardware area. The Manhattan distance is expressed by Equation (4) [17.

$$
M D(x, y)=\sum_{i=1}^{d}|S(i)-C(i)|
$$

Here is an example that demonstrates how the MD is calculated, suppose two pixel values are represented in binary format, the 
Manhattan distance between them is computed using equation (4), where $\mathrm{S}(\mathrm{i})$ and $\mathrm{C}(\mathrm{i})$ correspond to the $\mathrm{i}^{\text {th }}$ projected dimension which should contain q bits. Furthermore, the difference between two q-bit codes of each dimension should be measured with decimal distance. For instance let us consider q equals two then the MD is computed as follows:

$$
\begin{aligned}
M D(000100 ; 110000) & =d d(00 ; 11)+d d(01 ; 00)+d d(00 ; 00) \\
& =3+1+0=4
\end{aligned}
$$

Table 2. : Comparison Between Different Intra Prediction Algorithms

\begin{tabular}{ccccc}
\hline QP & Algorithm & PSNR (dB) & Comp.Ratio(\%) & Bit Rate (kbps) \\
\hline 20 & SAD & 43.8 & 11 & 338 \\
20 & SHD & 43.8 & 10 & 371.25 \\
20 & MD & 43.8 & 10 & 371.25 \\
20 & SSD & 43.7 & 9 & 412.5 \\
\hline 25 & SAD & 40.5 & 16 & 232 \\
25 & SHD & 40.4 & 15 & 247.5 \\
25 & MD & 40.5 & 15 & 247.5 \\
25 & SSD & 40.4 & 14 & 265.17 \\
\hline 30 & SAD & 37.1 & 25 & 149 \\
30 & SHD & 36.9 & 22 & 168.75 \\
30 & MD & 37 & 22 & 168.75 \\
30 & SSD & 36.6 & 21 & 176.78 \\
\hline 35 & SAD & 33.7 & 35 & 106 \\
35 & SHD & 33.6 & 32 & 116.01 \\
35 & MD & 33.8 & 31 & 119.75 \\
35 & SSD & 33.3 & 30 & 123.75 \\
\hline 40 & SAD & 31.4 & 44 & 84 \\
40 & SHD & 31 & 40 & 92.81 \\
40 & MD & 30.7 & 42 & 88.39 \\
40 & SSD & 30.1 & 39 & 95.19 \\
\hline & & & &
\end{tabular}

\section{PERFORMANCE EVALUATION OF DIFFERENT INTRA PREDICTION ALGORITHMS}

Here, a comparison between different intra prediction algorithms (SSD,SHD,MD) as well as the SAD algorithm utilized in [18] is presented. All of these algorithms utilize the vertical, horizontal and DC modes. The Mother-Daughter CIF video which consists of 300 frames is encoded with the Henson [18] H.264 encoder while changing the intra prediction algorithm and the Quantization Parameter (QP).

Table 2 shows the comparison results between SSD, SHD and MD algorithms versus (SAD) standard [18]. The quantization parameter varies from 20 to 35 with a step of 5 . It is pretty clear that the SAD algorithm outperforms the other algorithms from the PSNR, compression ratio and bit rate perspectives.

\section{PROPOSED INTRA PREDICTION APPROACH}

In this section the authors illustrate the proposed new technique that enhances the compression capability, PSNR and the bit rate of the H.264 encoder. Moreover, the hardware architecture of the proposed technique is presented.

It has been shown from the statistics that the vertical, horizontal, and DC prediction modes are frequently used than other modes. These modes imply higher correlation between the reference samples and the pixels to be predicted [5].

This fact inspires the authors to design the BPMM which combines the vertical, horizontal and DC modes in a single new intra $4 \times 4$

\begin{tabular}{|c|c|c|c|c|c|c|}
\hline M & U0 & $\mathrm{U} 1$ & $\mathrm{U} 2$ & U3 & [U0 $\quad$ U1 $\quad$ U2 $\quad$ U3] & [M] \\
\hline LO & $\mathbf{P 0 , 0}$ & $\mathbf{P 0}, 1$ & $\mathbf{P} 0,2$ & $\mathbf{P} \mathbf{0 , 3}$ & \multirow[t]{2}{*}{$1 \times 4$ vertical matrix } & $1 \times 1$ upper corner matrix \\
\hline L1 & $\mathbf{P 1 , 0}$ & $\mathbf{P 1 , 1}$ & $\mathbf{P} 1,2$ & $\mathbf{P 1}, 3$ & & \\
\hline $\mathbf{L 2}$ & $\mathbf{P} 2, \mathbf{0}$ & $\mathbf{P 2 , 1}$ & $\mathbf{P} 2, \mathbf{2}$ & $\mathbf{P} 2,3$ & {$\left[\begin{array}{llll}L & \text { L1 } & \text { L2 } & \text { L3 }\end{array}\right]$} & [Avs \\
\hline $\mathrm{L3}$ & $\mathbf{P 3}, \mathbf{0}$ & $\mathbf{P 3}, 1$ & $\mathbf{P 3}, \mathbf{2}$ & $\mathbf{P 3}, \mathbf{3}$ & 1x4 horizontal matrix & 1x1 DC matrix \\
\hline
\end{tabular}
prediction mode.

Fig. 3: Construction matrices

The best prediction matrix of the BPMM is calculated by trade-offs between values of the following four matrices: vertical, horizontal, DC and upper-left corner matrices. Figure 3 shows the construction matrix which consists of the aforementioned four matrices.

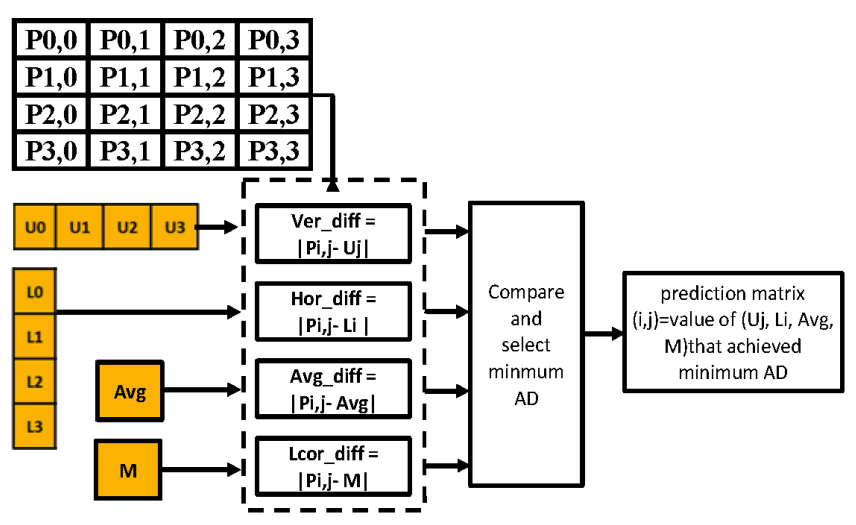

Fig. 4: The proposed intra prediction technique

Figure (4) illustrates the new proposed intra prediction technique. $P_{i, j}$ denotes the pixel to be predicted in the $i^{t h}$ row and $j^{\text {th }}$ column of current $4 \times 4$ block where $0<=i, j<=3, U_{j}$ and $L_{i}$ denote the reference samples reconstructed from upper and left blocks respectively. Algorithm 1 summarizes the procedure of generating the best prediction matrix.

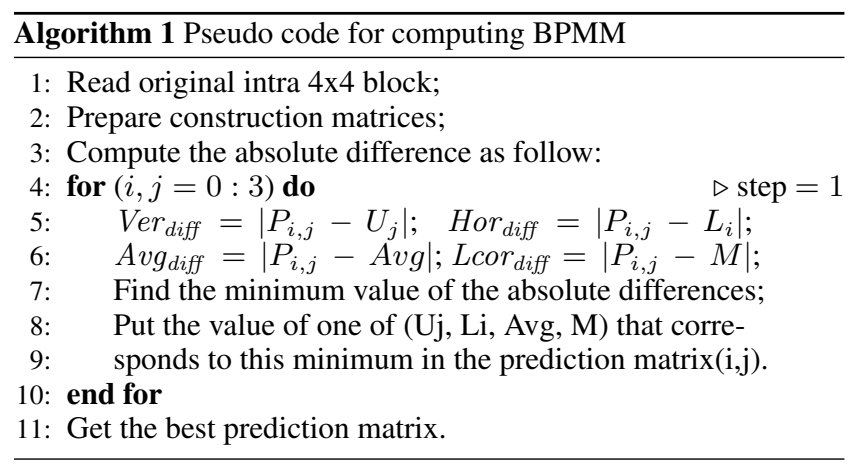




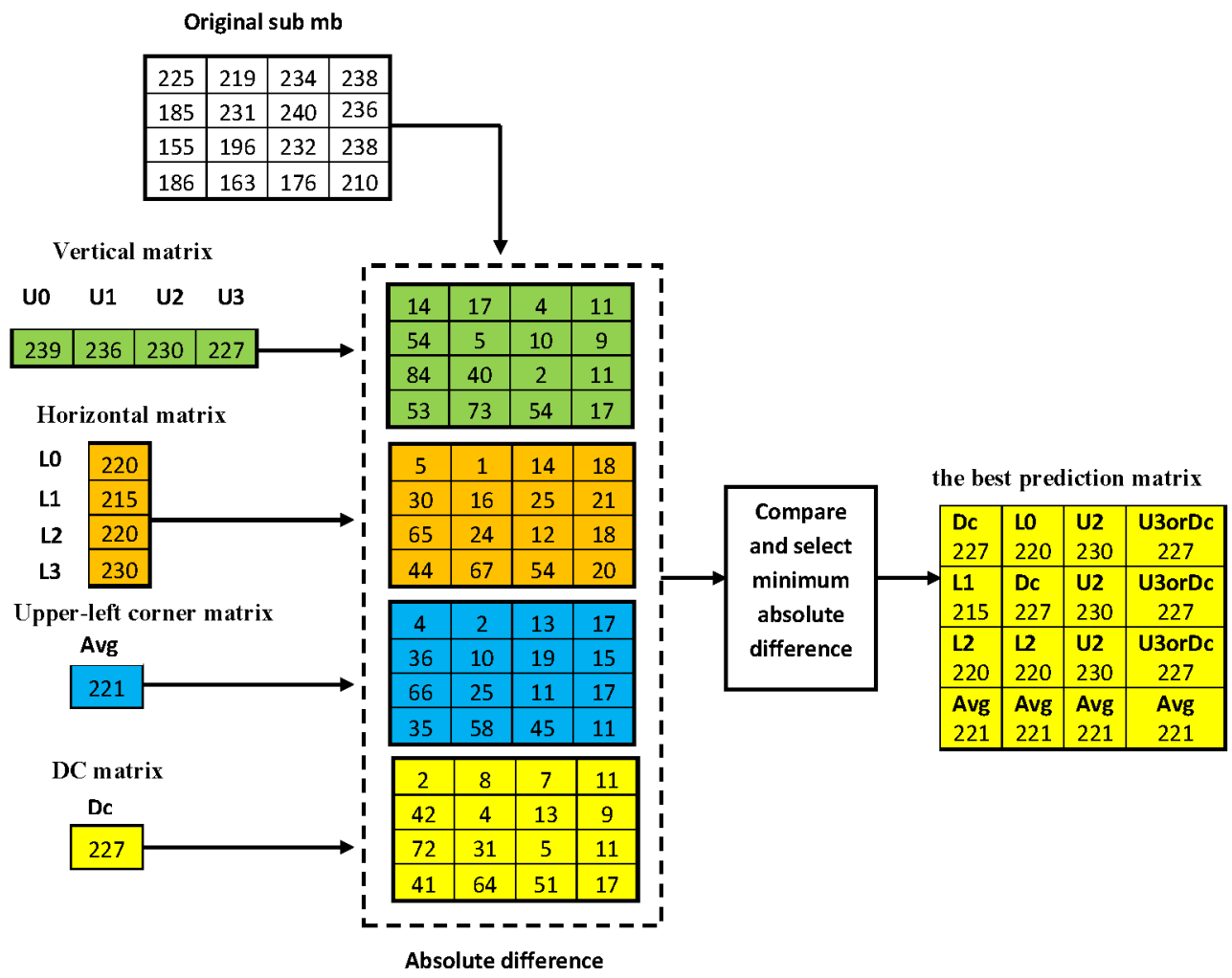

Fig. 5: Numerical example of applying the BPMM

Figure (5) demonstrates the proposed intra prediction technique by a numerical example. While Fig. (6) shows the

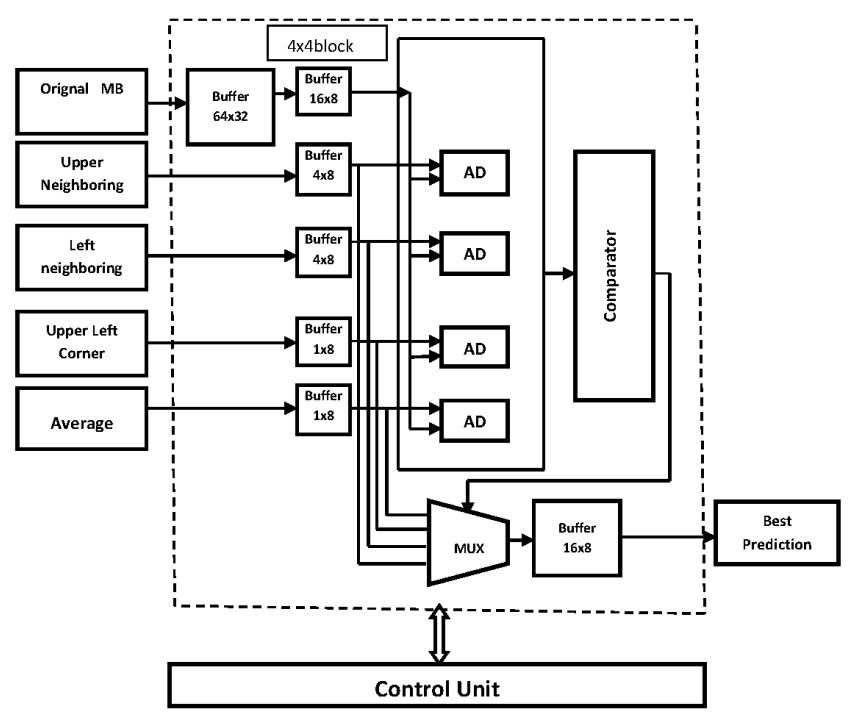

Fig. 6: Hardware architecture of the BPMM

hardware architecture that implements the intra prediction
approach(BPMM). This architecture is implemented using three main modules namely; intra prediction buffers, absolute difference units and a comparator.

Intra prediction buffer modules include six buffers. One buffer for storing original $\mathrm{MB}$, another buffer for storing original $4 \times 4$ block, and the four remaining buffers for storing the reconstructed pixel values which are needed to generate the predicted sub block (upper, left, upper-left corner, and average). The absolute difference module is responsible for calculating the absolute difference values. Finally the comparator module finds the minimum value of the absolute differences, then select a value between (upper, left, upper-left corner and average) that corresponds to this minimum and store it in the best prediction matrix.

\section{EXPERIMENTAL RESULTS}

In this section, the experimental setup as well as the results are explored. The new intra prediction technique is implemented in VHDL. The H.264 hardware encoder of Henson [18] is used as the basis of testing the new intra prediction approach.

\subsection{H.264 Encoder Hardware Testbed}

In this section, the hardware testbed of the H.264 encoder that has been employed to test the new proposed intra prediction technique is illustrated. The authors utilize the H.264 encoder testbed of Henson [6] that has been implemented using VHDL. This testbed is designed as a modular system with small, efficient, 
low power components doing well defined tasks. It exploits the intra prediction but it does not consider the inter prediction. It utilizes the Sum of Absolute Differences (SAD) algorithm while employing only the vertical, horizontal and DC prediction modes.

Video sequences of CIF format which consist of $352 \times 288$ pixels are used as input to the H.264 encoder testbed. Each frame of the input video sequence is divided into $396 \mathrm{MBs}$. Each MB consists of $256(16 \times 16)$ pixels while each pixel is represented by 8 bits. Each MB is transferred to the encoder testbed four pixels at a time using the 32 bits wide bus. The least significant byte of this wide bus is considered as the first pixel. The input video data is read in raster scan order then each $\mathrm{MB}$ is divided into $4 \times 4$ sub MB.

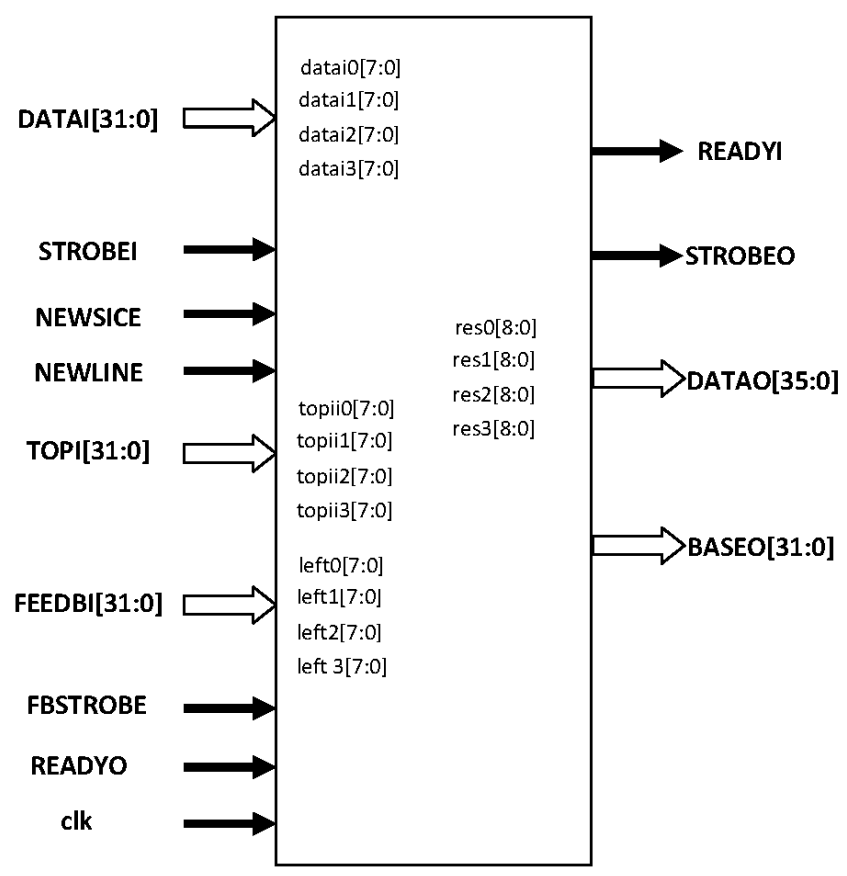

Fig. 7: Intra prediction block

The architecture of the intra prediction block is presented in Fig. (7). Before reading the first macroblock in a line, "NEWLINE" is set, this initializes all pointers and resets the prediction mode to no adjacent blocks. "NEWLINE" should be set for at least one CLK before "STROBEI" goes high. If this is the first in a slice, "NEWSLICE "should be set at the same time as newline. "STROBEI "and "READYI"are a handshaking signals. When READYI signal is high, request is sent to ram that hold YUV vidoe to output luma(Y) pixels values. "Datai" is the luma (Y) pixel values from ram which is of 32 bit length. The luma (Y) pixel values are validated by "STROBEI" signal. When this signal is high, luma pixel values are considered as valid values.

The main function of the intra prediction block is to create the residual values. The Residual values are the difference between original pixel values and the predicted pixel values. These residual values are applied to Transform, Quantization, Inverse Quantization and Inverse Transform (TQIQIT) blocks to get residual reconstructed pixel values. "TOPI" is the upper reconstructed pixel values , and "FEEDBI" is the left reconstructed pixel values which form input to Intraprediction block. The validity of "TOPI" and "FEEDBI" are ascertained by "FBSTROBE" signal with high status. Intra prediction block outputs reconstructed pixels through "BASEO". "DATAO" is the residual values that are the difference between original pixel values and predicted pixel values. These residual values are applied to TQIQIT module to get "TOPI" and "FEEDBI" pixel values. "READYO" and "STROBEO" are handshaking signals that responsible for organizing output data and receive reconstructed pixels.

\subsection{Performance Evaluation of the proposed Approach}

In this section, The authors present the experimental results of the new proposed algorithm compared to the SAD algorithm [18]. The ModelSim SE v.6.2 is utilized for the simulation. The comparison is based on three CIF videos namely Mother-Daughter, Container and Foreman which are composed of 300 frames. The evaluation process is accomplished as follow: each video is encoded with H.264 intra frame coder while utilizing the new BPMM. The quantization parameter varies from 20 to 45 with a step of 5 .

Figure (8) shows the waveforms for BPMM. "pred" is the best prediction matrix. "res0", "res1", "res2", "res3 "are the residual values generated by the difference of original pixels "datai0",to "datai3" and the best prediction matrix. for instance, subMB number 7 starts The intra prediction process at $3965 \mathrm{~ns}$ and continues up to 4095ns as shown in Fig. (9).

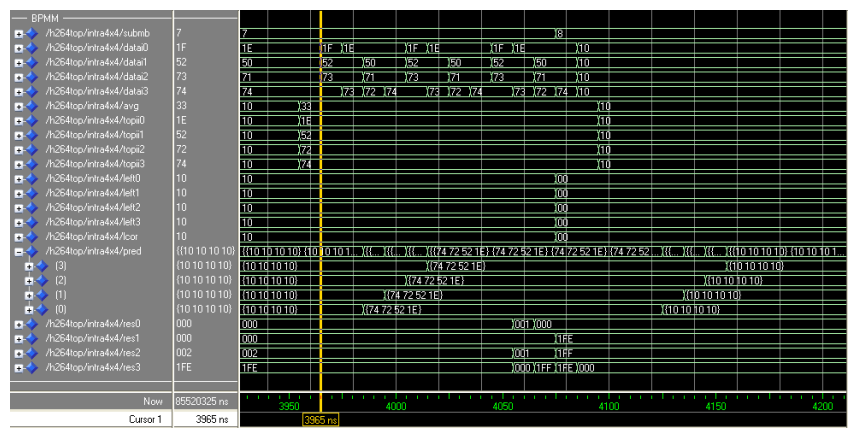

Fig. 8: Waveforms for simulating BPMM: Start of intra prediction for subMB \#7

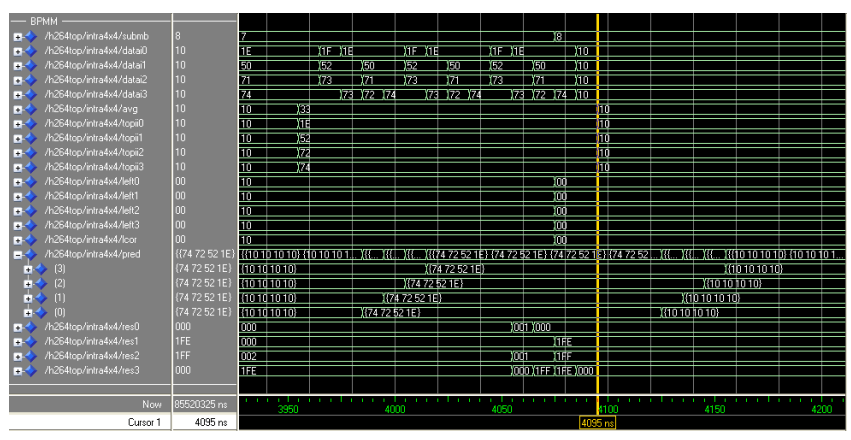

Fig. 9: Waveforms for simulating BPMM: End of intra prediction for subMB \#7 

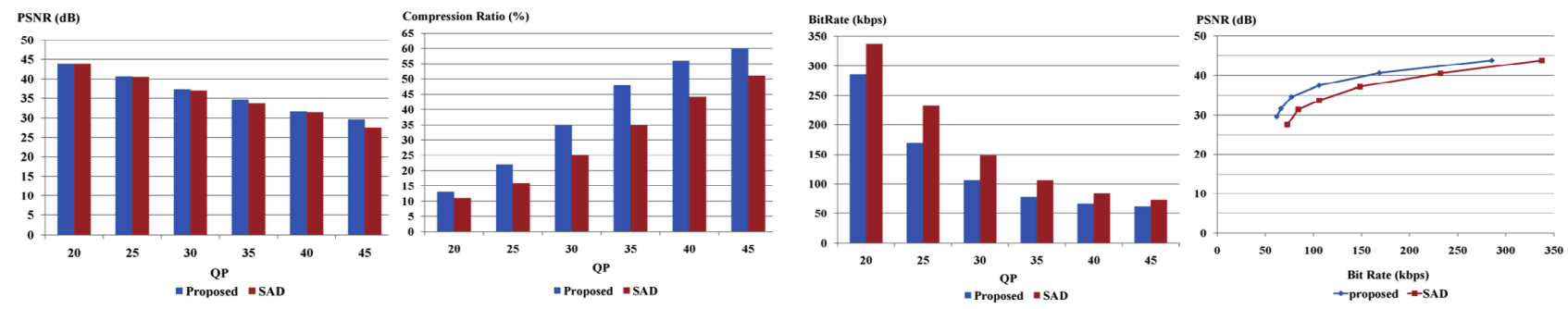

Fig. 10: Evaluation of BPMM using Mother-Daughter CIF video
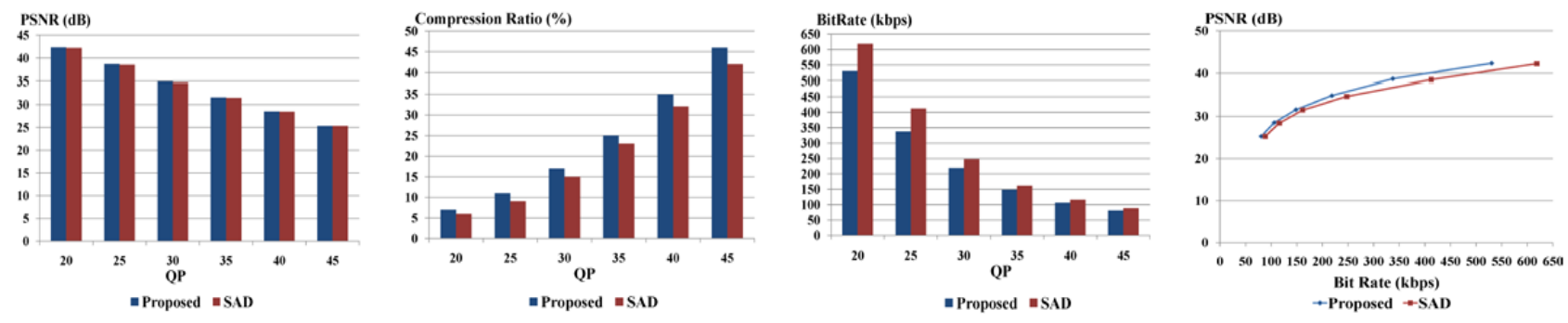

Fig. 11: Evaluation of BPMM using Container CIF video

Figures [10, 111 and (12) show the evaluation results of the new algorithm compared to the SAD. The results show that it enhances the compression ratio on average by $28.24 \%$ and consequentially the bit rate decreases on average by $22.5 \%$ while the PSNR is slightly increased on average by $2 \%$ for the Mother-Daughter CIF video.

Seeking for much more accurate judgement of the new proposed prediction technique the authors test it with the Container and Foreman CIF video. The results indicate that it enhances the compression ratio on average by $13.3 \%$ and correspondingly the bit rate decreases on average by $13.14 \%$ while the PSNR is slightly increased on average by $0.33 \%$ for the Container CIF video

Regarding the Foreman CIF video, the results indicate that the new prediction scheme enhances the compression ratio on average by $34.86 \%$ and correspondingly the bit rate decreases on average by $25.51 \%$ while the PSNR is slightly increased on average by $1.77 \%$. The pre-mentioned results have been achieved while maintaining the same execution time of the H.264 encoder using SAD.

\section{CONCLUSION}

Recently, video compression becomes very hot research topic due to the tremendous increase in video sizes and the limitations of the available bandwidth for transferring data. Among several video encoding standards H.264 is the most recent and efficient one. The intra prediction stage in the H.264 significantly contributes to its compression efficiency. In this paper, a new efficient H.264 intra $4 \times 4$ prediction scheme that relied on the combination of the most utilized intra prediction modes is proposed. First, several intra prediction algorithms were compared. The results show that the SAD algorithm outperforms others. Hence, it has been utilized as a reference algorithm to be compared with the proposed prediction scheme. Second, a performance evaluation of the new intra prediction scheme is accomplished. The results indicate that the new intra prediction scheme so-called BPMM achieves better compression ratios, bit rates and PSNR for the two CIF videos and over all the QP values under test. While it maintain the same execution time of the H.264 encoder.

\section{REFERENCES}

[1] S.H. Bharathi and Nagabhushana K. Raju. Verilog realization of Diagonal-Down-Left intra prediction for H.264 Video Encoder. International Journal of Engineering Research \& Technology (IJERT), 1(5):1-13, July 2012.

[2] I. Hamzaoglu, O. Tasdizen, and E. Sahin. An efficient h.264 intra frame coder system. IEEE Transactions on Consumer Electronics, 54(4):1903-1911, November 2008.

[3] Vimal Kumar Shrivastava, P. Muralidhar, and C. B. Rama Rao. Architecture for H.264 Intra Prediction Fast Mode Decision Algorithm. International Journal of Computer Applications, 68(3):1-6, April 2013.

[4] Bing Luo and Liyun Zhang. Fast Intra-prediction Mode Selection Method for H.264 Video Coding. In proceedings of the IEEE International Conference on Intelligent System Design and Engineering Application, volume 01 of ISDEA'10, pages 721-724, 2010.

[5] Liping Wang, Lai-Man Po, Yusuf Md. Salah Uddin, Ka-Man Wong, and Shenyuan Li. A Novel Weighted Cross Prediction for H.264 Intra Coding. In proceedings of the IEEE International Conference on Multimedia and Expo, ICME'09, pages 165-168, New York, NY, USA, 2009. IEEE Press.

[6] Tajdid Ul Alam, Jafor Ikbal, and Touhid Ul Alam. Fast DC Mode Prediction Scheme For Intra 4x4 Block In H.264/AVC Video Coding Standard. International Journal of Advanced Computer Science and Applications (IJACSA), 3(9):90-94, 2012.

[7] Daniel Palomino, Guilherme Corrêa, Claudio Diniz, Sérgio Bampi, Luciano Agostini, and Altamiro Susin. Algorithm 

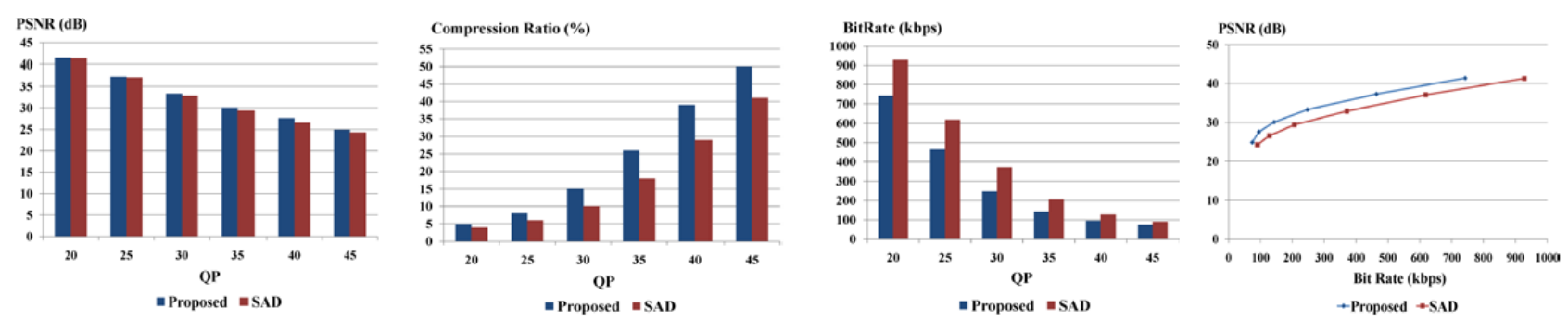

Fig. 12: Evaluation of BPMM using Foreman CIF video

and Hardware Design of a Fast Intra-Frame Mode Decision Module for H.264/AVC Encoders. In proceedings of the $24^{\text {th }}$ Symposium on Integrated Circuits and Systems Design, SBCCI' 11, pages 143-148, Pessoa, Brazil, 2011. ACM.

[8] Nejmeddine Bahri, Imen Werda, Amine Samet, Mohamed Ali Ben Ayed, and Nouri Masmoudi. Fast Intra Mode Decision Algorithm for H264/AVC HD Baseline Profile Encoder. International Journal of Computer Applications, 37(6):8-13, January 2012.

[9] Abderrahmane Elyousfi, Ahmed Tamtaoui, and EL-Houssine Bouyakhf. Fast Mode Decision Algorithm for Intra prediction in H.264/AVC Video Coding. International Journal of Computer Science and Network Security (IJCSNS), 7(1):356-364, January 2007.

[10] Y. N. Sairam, Nan Ma, and Neelu Sinha. A Novel Partial Prediction Algorithm for Fast $4 \times 4$ Intra Prediction Mode Decision in H.264/AVC. In proceedings of the IEEE Data Compression Conference, DCC'08, pages 232-241, Snowbird, UT, 2008. IEEE Computer Society.

[11] Youn-Long Steve Lin, Chao-Yang Kao, Hung-Chih Kuo, and Jian-Wen Chen. VLSI Design for Video Coding: H.264/AVC Encoding from Standard Specification to Chip. Springer Publishing Company, Incorporated, 1st edition, 2010.

[12] Yung-Lyul Lee, Ki-Hun Han, Dong-Gyu Sim, and Jeongil Seo. Adaptive Scanning for H.264 Intra Coding. ETRI Journal, 28(5):664-667, October 2006.

[13] Dae-Yeon Kim, Dong-Kyun Kim, and Yung-Lyul Lee. Adaptive Scanning Using Pixel Similarity for H.264/AVC. IEICE Transactions on Fundamentals of Electronics, Communications and Computer Sciences, E90-A(5):1112-1114, may 2007.

[14] Xu Xiang and Duan Zhe-min. Research and Improvement of Selection Algorithm Based on the H.264 Intra prediction Model. In proceedings of the 4th IEEE International Conference on Computer Science and Information Technology, ICCSIT'2011, pages 45-50, Chengdu, China, June 2011. IEEE Press.

[15] Joaqun Olivares, Javier Hormigo, Julio Villalba, and Ignacio Benavides. Minimum Sum of Absolute Differences Implementation in a Single FPGA Device. In proceedings of the 14th International Conference on Field Programmable Logic and Application, volume 3203 of FPLA'04, pages 986-990, Leuven, Belgium, September 2004. Springer.

[16] Mohammed Golam Sarwer and Q. M. Jonathan Wu. Effective Video Coding for Multimedia Applications, volume 2011, open access Chapter 3:Improved Intra Prediction of
H.264/AVC, pages 3.1-3.18. InTech Open Access Company, April 2011.

[17] Weihao Kong, Wu-Jun Li, and Minyi Guo. Manhattan Hashing for Large-Scale Image Retrieval. In proceedings of the 35th International ACM SIGIR Conference on Research and Development in Information Retrieval, SIGIR'12, pages 45-54, Portland, Oregon, USA, 2012. ACM.

[18] Andy Henson. H.264 hardware encoder in vhdl. Technical report, Zexia Access Ltd., 2008. 